\title{
Plancherel's Theorem and integration without the lattice condition
}

By

\author{
Michael Leinert
}

In part 1, a simple proof of the Plancherel Theorem is given using integration without the lattice condition on a space of Fourier transforms with the functional $I(\hat{f})=f(e)$. In part 2 we compare the approaches to integration without the lattice condition in [1] and [2]. It is shown that the assumptions used and the $\mathscr{L}^{1}$ spaces constructed in [1] and [2] are essentially the same and are in fact identical for reasonable examples like the important one in part 3 of [1] and the example considered in part 1 below.

Let us recall some definitions and facts from [2], which we are going to use. Let $X$ be a non-void set, $\mathscr{E}$ a vector space of real functions on $X$ (not necessarily a lattice) and $I$ a Daniell integral on $\mathscr{E}$, that is: a positive linear functional on $\mathscr{E}$, which is continuous from below, i.e. which satisfies condition (3) of [2], p. 258:

$$
f \in \mathscr{E}, \quad f_{n} \in \mathscr{E}^{+}, \quad \sum_{1}^{\infty} f_{n} \geqq f \Rightarrow \sum_{1}^{\infty} I\left(f_{n}\right) \geqq I(f) .
$$

Here, $\mathscr{E}^{+}$denotes the set of all $g \in \mathscr{E}$ with $g \geqq 0$. On the set $\mathscr{P}$ of all functions $f: X \rightarrow[0, \infty]$ we define the functional $\bar{I}$ by

$$
\bar{I}(f)=\inf \left\{\sum_{1}^{\infty} I\left(f_{n}\right) \mid \quad f_{n} \in \mathscr{E}^{+}, \quad \sum_{1}^{\infty} f_{n} \geqq f\right\}
$$

and for arbitrary $f$ we set

$$
\|f\|=\bar{I}(|f|),
$$

where $|f|$ is the pointwise absolute value of $f$. We let $\mathscr{E}^{\prime}=\{g \in \mathscr{E} \mid\|g\|<\infty\}$ and define a function $f$ to be integrable $\left(f \in \mathscr{L}^{1}\right)$, if for every $\varepsilon>0$ there is $g \in \mathscr{E}$ ' with $\|f-g\|<\varepsilon$. The Daniell integral $I$ on $\mathscr{E}^{\prime}$ extends by \|\| -continuity to the integral $\int$ on $\mathscr{L}^{1}$. If condition (8) of [2], p. 261 is satisfied, that is if

$$
\int f=\|f\| \text { for } f \in\left(\mathscr{L}^{1}\right)^{+},
$$

then the Monotone Convergence Theorem holds.

1. Let $G$ be a locally compact Abelian group, $\mathscr{K}(G)$ the space of continuous complexvalued functions on $G$ with compact support, and $\hat{G}$ the dual group of $G$ with the weak* topology. Given a Haar measure $\lambda$ on $G$ we construct a Haar measure $\mu$ on $\hat{G}$ and prove 
Plancherel's Theorem. The Fourier transformation on $\mathscr{L}^{1} \cap \mathscr{L}^{2}(G, \lambda)$ extends to an isometric isomorphism of $\mathscr{L}^{2}(G, \lambda)$ onto $\mathscr{L}^{2}(\hat{G}, \mu)$.

It suffices to prove the theorem with $\mathscr{K}(G)$ instead of $\mathscr{L}^{1} \cap \mathscr{L}^{2}(G, \lambda)$. We use integration as described above (actually for complex functions, but this does not cause any problems). Let

$$
\begin{aligned}
X & =\hat{G} \\
\mathscr{E} & =\{\hat{f} \mid f \in \mathscr{D}\} \text { where } \mathscr{D} \text { is the linear span of } \mathscr{K}(G) * \mathscr{K}(G) \\
I(\hat{f}) & =f(e) \text { where } e \text { is the identity element of } G .
\end{aligned}
$$

The functional $I$ is linear. If $f \in \mathscr{D}$ with $\hat{f} \geqq 0$, by Krein-Milman we have $\langle f, p\rangle$ $=\int f p d \lambda \geqq 0$ for all normalized positive definite functions $p$, in particular $\left\langle f, h * h^{*}\right\rangle \geqq 0$ for all $h \in \mathscr{K}(G)$ with $h * h^{*}(e)=1$. So $f$ is positive definite, hence $f(e) \geqq 0$. This proves that $I$ is positive. Let us now show that $I$ is continuous from below. Let $\hat{f} \in \mathscr{E}, \hat{f}_{i} \in \mathscr{E}^{+}$with $\sum \hat{f}_{i} \geqq \hat{f}$. We may assume $\sum f_{i}(e)<\infty$, and it suffices to prove that $\sum f_{i}-f$ is positive definite. Since each $f_{i}$ is positive definite, the series $\sum f_{i}$ converges uniformly (but does not necessarily converge in $\mathscr{L}^{1}(G)$ ). Let $\varepsilon>0$. Since $K=\{x|| \hat{f}(x) \mid \geqq \varepsilon\}$ is compact, there is $n_{0} \in \mathbb{N}$ with $\sum_{1}^{n} \hat{f}_{i} \geqq \hat{f}-\varepsilon$ on $K$ for all $n>n_{0}$. By the definition of $K$, this last inequality also holds outside $K$. So we have

$$
\left\langle\sum_{1}^{n} f_{i}-f, \chi\right\rangle \geqq-\varepsilon \text { for all } n>n_{0}, \quad \chi \in \hat{G}
$$

hence

$$
\left\langle\sum_{1}^{n} f_{i}-f, h * h^{*}\right\rangle \geqq-\varepsilon \quad \begin{aligned}
& \text { for all } n>n_{0}, \quad h \in \mathscr{K}(G) \\
& \text { with } h * h^{*}(e)=1 .
\end{aligned}
$$

Since $h * h^{*}$ has compact support, the duality makes sense also for the infinite sum $\sum_{1}^{\infty} f_{i}$ and we obtain

$$
\left\langle\sum_{1}^{\infty} f_{i}-f, \quad h * h^{*}\right\rangle \geqq-\varepsilon \quad(\text { hence } \geqq 0)
$$

for all $h \in \mathscr{K}(G)$ with $h * h^{*}(e)=1$. So $\sum f_{i}-f$ is positive definite, in particular $\sum f_{i}(e) \geqq f(e)$. Hence $I$ is a Daniell integral.

Since $\mathscr{E}$ is linearly generated by its positive elements, every $f \in \mathscr{E}$ has finite norm $\|f\|$, so $\mathscr{E}^{\prime}=\mathscr{E}$.

Assertion 1. Every element of $\mathscr{K}(\hat{G})^{+}$can be approximated in $\|\quad\|$ by elements of $\mathscr{E}^{+}$. In particular, $\mathscr{K}(\hat{G}) \subset \mathscr{L}^{1}(X, \mathscr{E}, I)$.

Proof. a) $\mathscr{K}^{+}=\mathscr{K}(\hat{G})^{+}$can be approximated in $\|\quad\|$ by $\mathscr{K}^{+} \mathscr{E}^{+}$: Let $f \in \mathscr{K}^{+}$and $S$ be the support of $f$. For given $\varepsilon>0$, by Stone-Weierstraß there is $g \in \mathscr{E}^{+}$with 
$|g-1|<\varepsilon$ on $S$. We then have

$$
|f-f g| \leqq \varepsilon|f| \text {. }
$$

Since $f \in \mathscr{K}^{+}$has finite norm (it can be dominated by some $h \in \mathscr{E}^{+}$because of StoneWeierstraß), (1.1) implies that $\mathscr{K}^{+}$can be approximated in \|\| by $\mathscr{K}^{+} \mathscr{E}^{+}$.

b) $\mathscr{K}^{+} \mathscr{E}^{+}$can be approximated in \|\| by $\mathscr{E}^{+} \mathscr{E}^{+}$. This is clear since $\mathscr{K}^{+}$can be approximated in sup-norm by $\mathscr{E}^{+}$.

Assertion 2. Every element of $\mathscr{E}$ can be approximated in \|\| by elements of $\mathscr{K}$.

Proof. a) $\mathscr{E}$ can be approximated in \|\| by $\mathscr{E} \cdot \mathscr{E}:$

$\mathscr{D}$ has an approximate convolution identity consisting of self-adjoint functions $g$ with $\|g\|_{1}=1$. If $\hat{f} \in \mathscr{E}^{+}$, then

$$
\begin{aligned}
& |\hat{f}-\hat{g} \hat{f}|=\hat{f}-\hat{g} \hat{f}, \quad \text { so } \\
& \|\hat{f}-\hat{g} \hat{f}\|=\bar{I}(\hat{f}-\hat{g} \hat{f})=I(\hat{f}-\hat{g} \hat{f})=f(e)-g * f(e) \rightarrow 0 .
\end{aligned}
$$

b) $\mathscr{E} \cdot \mathscr{E}$ can be approximated in \|\| by $\mathscr{K} \mathscr{E} \subset \mathscr{K}$, since $\mathscr{E}$ can be approximated in sup-norm by $\mathscr{K}$.

By Assertions 1 and 2 the lattice $\mathscr{K}(\hat{G})$ is a dense subset of $\mathscr{L}^{1}$ and $\int f=\|f\|$ for $f \in \mathscr{K}(\hat{G})^{+}$. Hence $\int f=\|f\|$ for $f \in\left(\mathscr{L}^{1}\right)^{+}$, that is (8) in [2] holds (and $\mathscr{L}^{1}$ is a lattice satisfying Stone's condition). The restriction of the integral to $\mathscr{K}(\hat{G})$ is a translation invariant positive linear functional $\mu$ which is nonzero since otherwise $I$ would vanish on $\mathscr{E}$ by Assertion 2. The $\mathscr{L}^{1}$ space and integral constructed from $\mathscr{K}(\hat{G})$ and the Haar measure $\mu$ coincide with the original $\mathscr{L}^{1}$ space and integral constructed from $\mathscr{E}$ and $I$ because of Assertions 1 and 2 and the equality $\bar{\mu}=\bar{I}$. The inequality $\bar{\mu} \geqq \bar{I}$ is clear, the reversed inequality follows from the fact that every $f \in \mathscr{E}^{+}$is the increasing limit of functions in $\mathscr{K}(\hat{G})^{+}$, hence can be written in the form $f=\sum f_{n}$ with $f_{n} \in \mathscr{K}(\hat{G})^{+}$.

We have done a little more than we need. For the actual proof of the theorem we shall only use that the translation invariant functional $I$ is really given by integration with respect to the Haar measure $\mu$ and that the image of $\mathscr{K}(G)$ is dense in $\mathscr{L}^{2}(\hat{G}, \mu)$. So, if we want to be short, we can replace Assertion 2 and the following by an argument like this (which also gives us Assertion 2 as a by-product): If $f \in \mathscr{E}^{+}$and $f_{n} \in \mathscr{K}(\hat{G})^{+}$with $f=\sum_{1}^{\infty} f_{n}$, then $\left\|f-\sum_{1}^{k} f_{n}\right\| \leqq \sum_{k+1}^{\infty}\left\|f_{n}\right\|=\sum_{k+1}^{\infty} \int f_{n} \rightarrow 0$, hence $I(f)=\sum_{1}^{\infty} \int f_{n}$, and on the other hand $f \in \mathscr{L}^{1}(\mu)$ and $\int f d \mu=\sum_{1}^{\infty} \int f_{n}$ by the Monotone Convergence Theorem for $\mu$. So $I(f)=\int f d \mu$.

Proof of Plancherel's Theorem. For $g \in \mathscr{K}(G)$ we have $\|g\|_{2}^{2}=g * g^{*}(e)=I\left(|\hat{g}|^{2}\right)$ $\left.=\int|\hat{g}|^{2}\right) d \mu=\|\hat{g}\|_{2}^{2}$.

Since $\mathscr{K}(\hat{G})$ can be approximated in $\|\quad\|_{2}$ by $\mathscr{E}=\hat{\mathscr{D}}$ (proof as for Assertion 1 with $\|_{2}$ instead of \|\|$)$, the image $\widehat{\mathscr{K}(G)} \supset \hat{\mathscr{D}}$ is dense in $\mathscr{L}^{2}(\hat{G})$. 
2. In [1], König has chosen an approach to integration different from that of [2], but analogous results to his can be obtained in the setting of [2], some of them actually with the very same proof (for instance the main results Satz 2.4, Folgerung 2.5, Satz 2.6 of [1]). This is not astonishing, since the assumptions used as well as the $\mathscr{L}^{1}$ spaces constructed in [1] and [2] are essentially the same and are absolutely identical whën $\mathscr{E}^{\prime}=\mathscr{E}$ i.e. when every $f \in \mathscr{E}$ has finite norm.

a) Let us look at the assumptions used in [1] and [2] beside positivity and linearity of I. If for any function $f$ we let $f^{+}=\max \{f, 0\}$ and $f^{-}=(-f)^{+}$, the continuity condition (*) of [1], p. 449 is

$$
\bar{I}\left(f^{+}\right)=I(f)+\bar{I}\left(f^{-}\right) \text {for } f \in \mathscr{E}
$$

or equivalently

$$
\bar{I}\left(f^{+}\right) \leqq I(f)+\bar{I}\left(f^{-}\right) \text {for } f \in \mathscr{E}
$$

In [2], conditions (3) and (8) have been used. Condition (8) supposes the integral on $\mathscr{L}^{1}$ and hence supposes (3), the continuity of $I$ from below, but using (13) of [2], p. 263 we may write (8) in the form

$$
I^{\prime}(f)=\bar{I}(f) \text { for } f \in\left(\mathscr{L}^{1}\right)^{+}
$$

where the functional $I^{\prime}$ is defined by

$$
I^{\prime}(f)=\inf \left\{\sum_{0}^{\infty} I\left(f_{n}\right) \mid f_{0} \in \mathscr{E}, f_{n} \in \mathscr{E}^{+} \text {for } n \geqq 1, \sum_{0}^{\infty} f_{n} \geqq f\right\}
$$

for arbitrary $f$. Note that $I^{\prime}(g) \leqq \bar{I}(g)$ for all $g \in \mathscr{P}$. An equivalent formulation of $(G)$ is

$$
\begin{aligned}
& I^{\prime}(f)=\bar{I}(f) \text { for } f=\sum_{0}^{\infty} f_{n} \geqq 0 \quad \text { with } f_{0} \in \mathscr{E}^{\prime}, \quad f_{n} \in \mathscr{E}^{+} \text {for } n \geqq 1, \\
& \sum_{0}^{\infty} I\left(f_{n}\right)<\infty .
\end{aligned}
$$

The equivalence of $(G)$ and $\left(G_{1}\right)$ is due to the fact that for $g \in\left(\mathscr{L}^{1}\right)^{+}$the number $I^{\prime}(g)$ is the infimum of $I^{\prime}(f)$ where $f$ ranges over functions as in $\left(G_{1}\right)$ satisfying also $f \geqq g$.

Clearly $(G)$ and $\left(G_{1}\right)$ each imply $I^{\prime}(0)=0$ which is equivalent to (3) in [2]. So instead of the two conditions (3) and (8) in [2] we can use condition $(G)$ (or condition $\left(G_{1}\right)$ ). However it may be difficult to verify $(G)$ or $\left(G_{1}\right)$ directly. In the above proof of the Plancherel Theorem for instance, it was quite convenient first to prove (3) and then to obtain $(G)$ i.e. $(8)$ along the way.

Let us now prove that, in the case $\mathscr{E}^{\prime}=\mathscr{E}$, the assumptions made in [1] and in [2] are identical.

Proposition. If $\mathscr{E}^{\prime}=\mathscr{E}$, then $(*)$ and $\left(G_{1}\right)$ are equivalent.

P r o o f. (i) It has been shown in [1], 1.8 that $(*)$ implies the property

$$
I^{\prime}(f)=\bar{I}(f) \text { for all } f \in \mathscr{P} .
$$


The converse implication $(K) \Rightarrow(*)$ is immediate (since $I^{\prime}(f+g)=I(f)+I^{\prime}(g)$ for $f \in \mathscr{E}$ and any function $g)$ : for $f \in \mathscr{E}$ by $(K)$ we obtain $\bar{I}\left(f^{+}\right)=I^{\prime}\left(f^{+}\right)$ $=I^{\prime}\left(f+f^{-}\right)=I(f)+I^{\prime}\left(f^{-}\right)=I(f)+\bar{I}\left(f^{-}\right)$, hence $(*)$.

(ii) Clearly, $(K)$ implies $\left(G_{1}\right)$. Let us now show that, since $\mathscr{E}^{\prime}=\mathscr{E},\left(G_{1}\right)$ implies $(K)$. In what follows we suppose $f_{0} \in \mathscr{E}$ and $f_{k}, f_{k}^{\prime} \in \mathscr{E}^{+}$for $k \geqq 1$. For any $g \in \mathscr{P}$ we have by $\left(G_{1}\right)$

hence $(K)$.

$$
\begin{aligned}
I^{\prime}(g) & =\inf \left\{I^{\prime}\left(\sum_{0}^{\infty} f_{k}\right) \mid \sum_{0}^{\infty} f_{k} \geqq g\right\}=\inf \left\{\bar{I}\left(\sum_{0}^{\infty} f_{k}\right) \mid \sum_{0}^{\infty} f_{k} \geqq g\right\} \\
& =\inf \left\{\sum_{1}^{\infty} I\left(f_{k}^{\prime}\right) \mid \sum_{1}^{\infty} f_{k}^{\prime} \geqq \sum_{0}^{\infty} f_{k} \geqq g\right\} \geqq \bar{I}(g),
\end{aligned}
$$

The assumption $\mathscr{E}^{\prime}=\mathscr{E}$ in the Proposition is not superfluous since $\left(G_{1}\right)$ is a slightly weaker condition than $(*)$ :

Example. Let $X=(0, \infty), f_{0}=-\chi_{(0,1)}+\chi_{[1, \infty)}$ and $f_{k}=\chi_{[1 /(k+1), 1 / k)}$ for $k \geqq 1$. Let $\mathscr{E}$. be the space of finite linear combinations $\sum c_{k} f_{k}$, and for $f \in \mathscr{E}$ let $I(f)$ denote the Lebesgue integral of $f$ from 0 to 1 . A finite linear combination $\sum c_{k} f_{k}$ is in $\mathscr{E}^{+}$if and only if $c_{0}=0$ and $c_{k} \geqq 0$ for $k \neq 0$, it is in $\mathscr{E}^{\prime}$ if and only if $c_{0}=0$. By the properties of the Lebesgue integral, condition (3) of [2] is satisfied. Since $\mathscr{E}$ is a lattice, condition (6) and hence (8) of [2] holds. But condition (*) of [1] is not satisfied since $\bar{I}\left(f_{0}^{+}\right)=\infty \neq-1+1=I\left(f_{0}\right)$ $+\bar{I}\left(f_{0}^{-}\right)$.

It has been shown by König that the condition

$$
f \in \mathscr{E}, \quad \bar{I}\left(f^{-}\right)<\infty \Rightarrow \bar{I}\left(f^{+}\right)<\infty
$$

is the difference between $(*)$ and $\left(G_{1}\right)$ in the sense that $(*)$ is equivalent to $\left(G_{1}\right)$ together with $(D)$ [see $(2.1)$ below].

If in [2] we modify the definition of integrability replacing $\mathscr{E}$ by $\mathscr{E}$, the assumptions of [1] and [2] become identical: condition $(8)$ and hence $(G)$ then is equivalent to $\left(G_{2}\right)$ where $\left(G_{2}\right)$ is as $\left(G_{1}\right)$ but with $\mathscr{E} \prime$ replaced by $\mathscr{E}$. The proof of the Proposition above just shows that $(*)$ and $\left(G_{2}\right)$ are equivalent.

To finish this paragraph let us give a list of equivalent formulations of condition $(*)$ and a corresponding list for condition (3) (H. König, personal communication):

$$
(*) \leftrightarrow(K) \leftrightarrow\left(G_{2}\right) \leftrightarrow\left(K_{1}\right) \leftrightarrow\left(K_{2}\right) \leftrightarrow\left(K_{3}\right) \leftrightarrow\left(G_{1}\right)+(D)
$$

where $\left(K_{1}\right),\left(K_{2}\right),\left(K_{3}\right)$ are the conditions

$\left(K_{1}\right)$

$$
I^{\prime}\left(f^{+}\right)=\bar{I}\left(f^{+}\right) \text {for } f \in \mathscr{E}
$$

$\left(K_{2}\right)$ $\bar{I}(v)=I(f)+\bar{I}(u)$ for all $f \in \mathscr{E}$, $\left(K_{3}\right)$ $\bar{I}(v) \leqq I(f)+\bar{I}(u)$ for all $f \in \mathscr{E}$, $u, v \in \mathscr{P} \quad$ with $v=f+u$ $u, v \in \mathscr{P}$ with $v=f+u$.

Since it is easier, we do not reproduce the original proofs by König but derive things from what we already have done. The first two equivalences have been proved in the above Proposition (delete the assumption " $\mathscr{E}$ ' $=\mathscr{E}$ " there and write $\left(\mathrm{G}_{2}\right)$ instead of $\left(\mathrm{G}_{1}\right)$ ). The implication $(K) \Rightarrow\left(K_{1}\right)$ is evident; $\left(K_{1}\right) \Rightarrow(*)$ is immediate since $I^{\prime}(f+g)$ $=I(f)+I^{\prime}(g)$ for $f \in \mathscr{E}$ and any function $g$. For the same reason, $(K)$ implies $\left(K_{2}\right)$. The 
implications $\left(K_{2}\right) \Rightarrow\left(K_{3}\right)$ and $\left(K_{3}\right) \Rightarrow(*)$ are evident (for the last take $\left.v=f^{+}, u=f^{-}\right)$. It remains to show the last equivalence of $(2.1)$. The implications $(*) \Rightarrow(D)$ and $(K) \Rightarrow\left(G_{1}\right)$ are clear. Conversely, $\left(G_{1}\right)$ together with $(D)$ implies $\left(G_{2}\right)$ : If $f=\sum_{0}^{\infty} f_{n} \geqq 0$ with $f_{0} \in \mathscr{E}$, $f_{n} \in \mathscr{E}^{+}$for $n \geqq 1, \sum_{0}^{\infty} I\left(f_{n}\right)<\infty$, then $\bar{I}\left(f_{0}^{-}\right) \leqq \sum_{1}^{\infty} I\left(f_{n}\right)<\infty$, so by $(D) \bar{I}\left(f_{0}^{+}\right)<\infty$, hence $f_{0} \in \mathscr{E}^{\prime}$. So $f=\sum_{0} f_{n}$ is as in $\left(G_{1}\right)$ and we obtain $I^{\prime}(f)=\bar{I}(f)$ by $\left(G_{1}\right)$. This completes the proof of (2.1).

$$
(3) \leftrightarrow\left(c_{1}\right) \leftrightarrow\left(c_{2}\right) \leftrightarrow\left(c_{3}\right) \leftrightarrow\left(c_{4}\right)
$$

where (3) is as given in the introduction and the other conditions are

$\left(c_{1}\right) \quad f_{n} \in \mathscr{E}, \quad f_{n+1} \geqq f_{n}, \quad \lim f_{n} \geqq 0 \Rightarrow \lim I\left(f_{n}\right) \geqq 0$

$\left(c_{2}\right) \quad I^{\prime}(0)=0 \quad$ (that is $\left.I^{\prime}(0) \geqq 0\right)$

$\left(c_{3}\right) \quad I^{\prime}(f)=I(f) \quad\left(\right.$ that is $I^{\prime}(f) \geqq I(f)$ ) for $f \in \mathscr{E}$.

$\left(c_{4}\right) \quad I(f) \leqq \bar{I}\left(f^{+}\right)$for $f \in \mathscr{E}$.

Each of the conditions $\left(c_{i}\right)$ is easily seen to be equivalent to (3).

b) Let us look at the $\mathscr{L}^{1}$ spaces. It follows from the definitions in [1] and [2] and 2.1.(i), Satz 2.9, Satz 2.10 in [1] that $\mathscr{L}^{1}$ of [2] consists precisely of those $f$ in $\mathscr{L}^{1}$ of [1] that have $\|f\|<\infty$. This can also be seen directly:

Let the functional $I$ satisfy $(*)$. The fact that $I^{\prime}(f)+I^{\prime}(-f)=0$ for some $f: X \rightarrow \overline{\mathbb{R}}$ amounts to saying that for every $\varepsilon>0$ there are functions $f_{0}, g_{0} \in \mathscr{E}, f_{n}, g_{n} \in \mathscr{E}^{+}$for $n \geqq 1$ with

$$
-\sum_{0}^{\infty} f_{n} \leqq f \leqq \sum_{0}^{\infty} g_{n} \text { and } \sum_{0}^{\infty} I\left(f_{n}\right)+\sum_{0}^{\infty} I\left(g_{n}\right)<\varepsilon
$$

We then have

$$
\left|f-\sum_{0}^{k} g_{n}\right| \leqq \sum_{0}^{\infty} f_{n}+\sum_{0}^{\infty} g_{n}+\sum_{k+1}^{\infty} g_{n}
$$

hence $\left\|f-\sum_{0}^{k} g_{n}\right\|=I^{\prime}\left(\left|f-\sum_{0}^{k} g_{n}\right|\right)<2 \varepsilon$ for $k$ large enough. Conversely if for $\varepsilon>0$ there is $g \in \mathscr{E}$ with $\|f-g\|<\varepsilon$, it is clear how to obtain functions $f_{n}, g_{n}$ as in (2.3).

From this we see that the $\mathscr{L}^{1}$ spaces of [1] and [2] coincide if $\mathscr{E}^{\prime}=\mathscr{E}$. We can obtain equality of the $\mathscr{L}^{1}$ spaces in general, if we modify the definition of integrability in [2] replacing $\mathscr{E}^{\prime}$ by $\mathscr{E}$.

Let us summarize, taking into account also a):

(i) If $\mathscr{E}^{\prime}=\mathscr{E}$, the assumptions and the $\mathscr{L}^{1}$ spaces of [1] and [2] are the same. This applies, for instance, to the important example considered in part 3 of [1] and to the example above yielding Plancherel's Theorem.

(ii) We can achieve that the assumptions and the $\mathscr{L}^{1}$ spaces of [1] and [2] are the same in general by modifying the definition of integrability in [2] (using approximation by $\mathscr{E}$ instead of $\left.\mathscr{E}^{\prime}\right)$.

(iii) If $(*)$ is not satisfied, $\left(G_{1}\right)$ may still hold and so it may still be possible to work in the setting of [2]. This situation, however does not seem to occur in reasonable examples. 
Appendix. We note that, with a slight modification, part 1 can also be done using Riemann integration together with usual integration:

a) The general procedure of Riemann integration: Given a vector space $\mathscr{E}$ of real functions on $X$ and a positive linear functional $I$ on $\mathscr{E}$ (where no continuity assumption is made), define the Riemann norm \| $\|$ by

$$
\|f\|=\inf \left\{I(g)\left|\quad g \in \mathscr{E}^{+}, \quad g \geqq\right| f \mid\right\}
$$

and let $\mathscr{R}$ be the space of all Riemann integrable functions i.e. of all functions that can be approximated in $\|\quad\|$ by elements of $\mathscr{E}$. Since $|I(f)| \leqq\|f\|$ for $f \in E$, there is a unique continuous extension $\int$ of $I$ on the space $\mathscr{R}$ (the elements of norm $\infty$ are no obstacle to this). The integral $\int$ on $\mathscr{R}$ is positive, since every $f \in \mathscr{R}^{+}$can be approximated in $\|\quad\|$ by elements of $\mathscr{E}^{+}$:

If $f \in \mathscr{R}^{+}, g \in \mathscr{E}, h \in \mathscr{E}^{+}$with $|f-g| \leqq h$, then $|f-(g+h)| \leqq 2 h$ and $g+h \geqq 0$, so $g+h \in \mathscr{E}^{+}$.

The construction of $\mathscr{R}$ and $\int$ can also be done by means of upper and lower Riemann integral. The result is the same. Things can also be done starting from $\mathscr{E}_{0}$, the space of all $f \in \mathscr{E}$ with $\|f\|<\infty$. This makes a difference, but in the example we want to consider, it is the same.

b) Let $X, \mathscr{E}, I$ be as in part 1 . Assertions 1 and 2 still hold for the Riemann norm, in particular $\mathscr{K}(\hat{G}) \subset \mathscr{R}$ and on $\mathscr{K}(\hat{G})$ the integral defines a Haar measure $\mu$. For $f \in \mathscr{E}^{+}$ there is a sequence of functions $f_{n} \in \mathscr{K}(\hat{G})^{+}$with $\left\|f_{n}-f\right\| \rightarrow 0$ and $f_{n} \pi f$. Using the Monotone Convergence Theorem for the Haar measure $\mu$ we see that $f \in \mathscr{L}^{1}(\mu)$ and $I(f)=\int f d \mu$. By linearity, this holds for all $f \in \mathscr{E}$. Now the proof of Plancherel's Theorem is as in part 1. The \|\|$_{2}$ approximation at the end has to be done with respect to $\mu$ (in part 1 it could also be done with respect to $I$ ).

\section{References}

[1] H. KöNIG, Integraltheorie ohne Verbandspostulat. Math. Ann. 258, 447-458 (1982).

[2] M. Leinert, Daniell-Stone integration without the lattice condition. Arch. Math. 38, 258-265 (1982).

[3] W. Rudin, Fourier Analysis on Groups. New York-London-Sydney 1960.

Eingegangen am 12.11.1982*)

Anschrift des Autors:

Michael Leinert

Institut für Angewandte Mathematik

Universität Heidelberg

Im Neuenheimer Feld 294

D-6900 Heidelberg

*) Eine Neufassung ging am 5. 5.1983 ein. 